\title{
Therapeutics discovery: From bench to first in-human trials"
}

\author{
ENSAF M. AL-HUJAILY ${ }^{1 * *}$, TANVIR KHATLANI ${ }^{* * *}$, ZEYAD ALEHAIDEB $^{1}$, RIZWAN ALI $^{1}$, BADER ALMUZAINI $^{1}$, \\ BAHAUDDEEN M ALRFAEI ${ }^{1}$, JAHANGIR IQBAL ${ }^{1}$, IMADUL ISLAM ${ }^{1}$, SHUJA MALIK ${ }^{1}$, BADER A MARWANI ${ }^{1}$, \\ SALAM MASSADEH $^{1}$, ATEF NEHDI $^{1}$, BARRAK ALSOMAIE $^{1}$, BADER DEBASI $^{1}$, \\ IBRAHEEM BUSHNAK ${ }^{1}$, SAEED NOIBI ${ }^{2}$, SYED HUSSAIN ${ }^{3}$, WAHID ABDUL WAJID ${ }^{4}$, JEAN-PIERRE ARMAND ${ }^{5}$, \\ SHERAZ GUL $^{6}$, JULEN OYARZABAL ${ }^{7}$, RANA RAIS $^{8}$, CHAS BOUNTRA $^{9}$, AHMED ALASKAR $^{1}$, \\ BANDER AL KNAWY ${ }^{1}$ and MOHAMED BOUDJELAL ${ }^{1}$ \\ ${ }^{1}$ King Abdullah International Medical Research Center, King Saud bin Abdulaziz University for Health Sciences, \\ Ministry of National Guard Health Affairs, Riyadh, Al Hassa; ${ }^{2}$ GlaxoSmithKline, Jeddah, Kingdom of Saudi Arabia; \\ ${ }^{3}$ Plymouth University, Peninsula Schools of Medicine and Dentistry, Plymouth, UK; ${ }^{4}$ Process and Manufacturing Sciences, \\ Agenus Inc., Berkeley, CA, USA; ${ }^{5}$ Gustave Roussy, Cancer Campus Grand, Paris, France; \\ ${ }^{6}$ Fraunhofer Institute for Molecular Biology and Applied Ecology ScreeningPort, Hamburg, Germany; \\ ${ }^{7}$ Center for Applied Medical Research University of Navarra, 31008 Pamplona, Spain; \\ ${ }^{8}$ Drug Metabolism and Pharmacokinetics, Johns Hopkins Drug Discovery, Baltimore, MD, USA; \\ ${ }^{9}$ Structural Genomics Consortium, University of Oxford, Oxford, UK
}

Received December 15, 2017; Accepted January 10, 2018

DOI: $10.3892 / \mathrm{br} .2018 .1052$

Abstract. The 'Therapeutics discovery: From bench to first in-human trials' conference, held at the King Abdullah

Correspondence to: Dr Mohamed Boudjelal, King Abdullah International Medical Research Center, King Saud bin Abdulaziz University for Health Sciences, Ministry of National Guard Health Affairs, P.O. Box 22490, Riyadh 11426, Mail Code 1515, Kingdom of Saudi Arabia

E-mail: boudjelalmo@ngha.med.sa

*Proceedings of the conference; the conference and these proceedings are dedicated to the soul of Dr Sabhi Rahman, KAIMRC scientist and part of the conference team, who passed away while the preparations were ongoing

${ }^{* *}$ Contributed equally

Abbreviations: KAIMRC, King Abdullah International Medical Research Center; MNGHA, Ministry of National Guard Health Affairs; KSA, Kingdom of Saudi Arabia; USA, United States of America; UK, United Kingdom; SGC, Structural Genomics Consortium; SFDA, Saudi Food and Drug Administration; NCBE, National Committee of Bioethics; IRB, Institutional Review Board; MERS-CoV, Middle East Respiratory Syndrome; PCSK9, proprotein convertase subtilisin/kexin type 9; LDL, low-density lipoprotein; OASL, 2'-5'-oligoadenylate synthetase-like; CART, chimeric antigen receptor T-cell therapy; TIL, tumor-infiltrating T-cells; HRs, hormonal receptors; $\mathrm{CD}$, cluster of differentiation; $\mathrm{TNBC}$, triple-negative breast cancer

Key words: KAIMRC, MNGHA, clinical trials, phase I, CART, drug target
International Medical Research Center (KAIMRC), Ministry of National Guard Health Affairs (MNGHA), Kingdom of Saudi Arabia (KSA) from October 10-12, 2017, provided a unique opportunity for experts worldwide to discuss advances in drug discovery and development, focusing on phase I clinical trials. It was the first event of its kind to be hosted at the new research center, which was constructed to boost drug discovery and development in the KSA in collaboration with institutions, such as the Academic Drug Discovery Consortium in the United States of America (USA), Structural Genomics Consortium of the University of Oxford in the United Kingdom (UK), and Institute of Materia Medica of the Chinese Academy of Medical Sciences in China. The program was divided into two parts. A pre-symposium day took place on October 10, during which courses were conducted on clinical trials, preclinical drug discovery, molecular biology and nanofiber research. The attendees had the opportunity for one-to-one meetings with international experts to exchange information and foster collaborations. In the second part of the conference, which took place on October 11 and 12, the clinical trials pipeline, design and recruitment of volunteers, and economic impact of clinical trials were discussed. The Saudi Food and Drug Administration presented the regulations governing clinical trials in the KSA. The process of preclinical drug discovery from small molecules, cellular and immunologic therapies, and approaches to identifying new targets were also presented. The recommendation of the conference was that researchers in the KSA must invest more fund, talents and infrastructure to lead the region in phase I clinical trials and preclinical drug discovery. Diseases affecting the local population, such as Middle East Respiratory Syndrome and resistant bacterial infections, represent the optimal starting point. 


\section{Conference}

On behalf of the King Abdullah International Medical Research Center (KAIMRC) and Ministry of National Guard Health Affairs (MNGHA), Dr Bandar Alknawy, Chief Executive Officer of the MNGHA, and Dr Ahmed Alaskar, Executive Director of KAIMRC, welcomed the speakers, presenters, and attendees to the conference. Dr Alknawy and Dr Alaskar stated that this event is in alignment with Saudi Arabia Vision 2030, the plan to reduce dependency on oil, diversify the economy, and develop public service sectors such as health, education, infrastructure, recreation and tourism in the Kingdom of Saudi Arabia (KSA). Accordingly, KAIMRC is leading a transformation in biomedical research in the KSA to convert basic research into tangible products that impact health and economy. Dr Alknawy and Dr Alaskar then stated the aims of the conference: to showcase the research discoveries of KAIMRC and MNGHA with the intention of creating a platform and opportunity for exchange and collaboration between local and international researchers on therapeutics discovery and phase I clinical trials.

The opening ceremony was followed by a short presentation of the different sessions making up the scientific program of this 2-day event by the Conference Chairman, Dr Mohamed Boudjelal.

\section{Conference first day}

On the first day of the conference, the presentations focused on the settings, regulation, and ethical aspects of phase I clinical trials. In addition, several international and KAIMRC clinical trials programs were presented, followed by round table discussions to provide expert recommendations. The program of the first day was divided into sessions as summarized in the following sections:

Session 1: Setting up a phase I clinical trials unit. The conference was opened by Professor Chas Bountra [Chief Scientist, Structural Genomics Consortium (SGC), University of Oxford, UK], who gave a presentation entitled 'Therapy Discovery in Academia vs. Biotech and Pharma'. Professor Bountra emphasized that the discovery of new medicines is too risky as in most cases the drug targets are not well validated and the disease biology is not well understood that lead to poor translation from animal to human, in addition the process is slow and costly. There is massive duplication and waste in biomedical research, a lack of reproducibility of published work, and in many increasingly important therapeutic areas, we are simply not producing what society and patients want. The validation of pioneer targets for drug discovery remains a major challenge. Professor Bountra gave an overview of how the SGC is overcoming these challenges by doing the following: i) Working with a large number of pharmaceutical companies to develop high-quality small-molecule inhibitors using structure-based drug design; and ii) focusing only on novel targets or those deemed 'difficult' or 'intractable', and giving these inhibitors to a large and growing international network of academic collaborators to crowd source new biology, disease understanding, and 'target discovery'.

The SGC has already facilitated proprietary efforts in pharma, catalyzed the creation of new biotech, and acceler- ated numerous clinical studies. Based on these achievements, the SCG is now: i) Generating 'Target Enabling Packages' (comprising purified proteins, biophysical or biochemical assays to assess function, three-dimensional X-ray structures, chemical starting points, Clustered Regularly Interspaced Short Palindromic Repeats genome editing reagents and antibodies) for novel, high-priority, disease-linked genes; ii) Guiding the production of platforms of primary human cells for the screening of novel inhibitors to identify new, 'better' targets for drug discovery; and iii) building major collaborations with patient groups, key clinicians, hospitals, and large academic drug-discovery centers to catalyze more clinical 'proof-of-concept' studies.

Professor Bountra ended his talk by stating that 'Together we are creating a new ecosystem for drug discovery. One which we believe will accelerate the generation of more novel 'precision' medicines, quickly. We hope these efforts will also reduce parallel clinical studies in patients with targets destined for failure, and will help generate more affordable medicines'.

The second talk, delivered by Dr Omar Abdulhameed Al Mazroo [Clinical Trials Consultant, Drug-Vice President, Saudi Food and Drug Administration (SFDA), KSA], was entitled 'Phase I Clinical Trials in the Kingdom of Saudi Arabia: Are We Ready?' Dr Al Mazroo summarized the readiness of the KSA to carry out phase I clinical trials. He explained that the main aims of research for all new products are to prove their safety and efficacy profiles while maintaining their qualities. The process of developing new pharmaceutical products consists of testing new chemical entities in many different stages, a process that is also applicable to biologics. Pharmaceutical companies run numerous in vitro, ex vivo and in vivo tests/trials as part of preclinical studies, and each regulatory authority establishes minimum requirements for the submission of these studies. It is essential to have enough animal data as prerequisite information before going to the first in-human phase I clinical trials. However, in the past, some first in-human phase I clinical trials have had disastrous consequences that put patients' safety in jeopardy. Subsequently, more stringent laws and regulations were developed, updated, released and enforced. Based on the Saudi Vision 2030 plan and because of the huge market share of the pharmaceutical industry, it has been the focus of the local authorities to attract and facilitate the establishment of new pharmaceutical companies, manufacturing sites, and research centers. The SFDA has become a leading regulatory body in the Gulf Cooperation Council area. Dr Al Mazroo presented the current thinking of the SFDA regarding setting up local clinical research activities and publishing guidelines. In addition, the 'Saudi Clinical Trials Registry' was launched to organize and regulate clinical research activities. Currently, the priority of the SFDA is to provide clear regulations and guidelines for the first in-human clinical trials based on current global practice.

This session concluded with a talk entitled 'Personalized Medicine and Phase I Trials' by Professor Jean-Pierre Armand (Senior Consultant Oncology, Intitut Gustave Roussy, Cancer Campus Grand, Paris, France). Professor Armand highlighted the advances in molecular oncology and cancer genetics made in the last 20 years that have defined many of the key oncogenes driving human cancer. Most common cancers are currently being revisited on the basis of modern molecular 
portraits that allow the identification of new molecular subtypes and therapeutic opportunities. These promise greater clinical efficacy and fewer adverse effects compared with traditional chemotherapeutic agents, and include inhibitors of the BCR-ABL (imatinib), Epidermal growth factor receptor (gefitinib), and BRAFV600E (vemurafenib) protein kinases or CYP17 inhibitors, which have transformed the treatment of chronic myelogenous leukemia, non-small cell lung cancer, melanoma, and prostate cancers, respectively. Molecular medicine programs aim to provide targeted therapies taking into account patient-specific tumor genomic profiles. Several clinical trials are ongoing to evaluate the concept of personalized and molecular medicine. These trials will use genomic and proteomics approaches to discover sensitive molecular-targeted therapies and develop an adequate molecular characterization of each patient's cancer. Professor Armand then gave an example of a clinical trial being carried out at the Institut Gustave Roussy. Molecular screening for cancer treatment optimization is a prospective molecular triage trial designed to identify actionable targets and match them with the selected targeted therapy. The final objective of this trial is to improve outcomes. The paradigm shift from the cancer cell as the main target to immunocompetent cells highlights the fact that, more than ever, the acid test of phases I/II is a critical moment in drug development. With the poor relevance of animal studies and complexity of immune combination, translational research will constitute a major step during phase I. Professor Armand summarized his talk by stating that characterization of the genomic and immune changes that drive an individual patient's disease is critical to inform rationally targeted therapies and treatment planning for patients with advanced cancer.

Session 2: Management of phase I clinical trials. A structured, practical approach to trial management is key to successful clinical trials: Many have failed to deliver drugs to the market as they did not implement effective techniques and management systems. In addition, the successful execution of a clinical trial requires a team of experts, strong collaboration, careful planning, coordination and management. One of the most important elements of successful translational research is to have basic scientists with expertise in the fundamental biology and molecular mechanisms of the disease under investigation. These scientists need to work with clinical researchers, who have a clinical understanding of human health and disease. The introduction of new technologies in the world of precision medicine may significantly reduce the duration of clinical trials. Finally, understanding the ethical precepts and regulations behind the trial design is also essential to a successful clinical trial. The session speakers covering these topics included: Professor Syed Hussain (Chair in Oncology Plymouth University, Peninsula Schools of Medicine and Dentistry, Plymouth, UK), Dr Jordi Rodon (Consultant, MD Anderson's Investigational Cancer, USA), Professor Robert Hawkins (Chair of Medical Oncology, University of Manchester and Christie Hospital, UK), and Professor Muhammad Z. Alkawi (Director of Ethic Office; King Abdulaziz City for Science and Technology, KSA).

Professor Sayed Hussain's presentation was entitled 'Early Phase to Late Phase Clinical Trials: From Design to Delivery'. Professor Hussain discussed the importance of infrastructure, teamwork, research facilities, and strong collaboration for the success of drug development from the initial design to final delivery. He addressed the need for a strong 'Clinical Research Unit', 'Pharmacology Unit' and 'Good Clinical Laboratory Practice Laboratory Facilities' for successful translational research during the early to late phases of clinical trials. In his presentation, Professor Hussain suggested that biorepositories play a key role in the reproducibility of clinical trials. Furthermore, he suggested that there is a need for an 'Early Drug Development Unit' that will improve the resources investment and strengthen the 'Accelerated Drug Development' program. He emphasized that the success of translational research is not only a function of the quality of the science, but also of the collaboration between academia and industry. Although academia and industry have substantial differences, they share the common goal of improving the health of patients. He alluded to KAIMRC Clinical Research Facility as an institution with great potential for development into a clinical research unit. However, he cautioned that drug development is expensive and time-consuming. Of thousands of compounds prescreened for drug testing, only a few become a drug on the market. Professor Hussain concluded that small molecular inhibitors are more feasible for drug development programs, especially given the current movement from a 'one-size-fits-all' approach to a personalized medicine strategy that focuses on biomarkers, molecular genetics, proteomics and metabolomics.

Dr Jordi Rodon gave a talk about incorporating precision medicine into phase I clinical trials. The talk was a brief overview of the role of genomics in early drug discovery. During his talk, Dr Rodon focused on the co-development of diagnostics and therapeutics, which has reduced the time from target discovery to drug approval. He emphasized that the molecular selection of patients is a driver of knowledge regarding the incorporation of precision medicine into early drug development. He stated that clinical trial design must continue to evolve to take advantage of this ever-expanding body of knowledge. Dr Rodon also gave credit to new technologies, such as next-generation sequencing, that have led to improvements in clinical research. He suggested the use of deep sequencing to understand the molecular mechanisms underlying the differences between responders and nonresponders during drug development. Dr Rodon mentioned that the objectives of phase I clinical trials have changed over time, so there is a need to remodel the objectives of phase I clinical trials: To move away from classical goals by creating a merger of phase I and II trials. This merger will lead to basket trials and larger phase I trials, the success of which will depend on the rigor of both preclinical development and clinical trial design. Basket trials are an emerging form of clinical trial that combine the classical clinical trial methodology with rapidly growing precision medicine. Precision medicine has the potential to generate a model for translational research in early drug development. Some of the key points to be considered while planning to incorporate precision medicine into phase I clinical trials are turnaround time, multiplexing, customizability, adaptability, and repeatability. Furthermore, laboratory evaluation, regulatory and ethical issues, record keeping, funding, and logistics are also essential ingredients for building a successful molecular prescreening program. 
Professor Robert Hawkins discussed the ethical aspects of clinical trials. Clinical research has expanded tremendously in the past few decades, necessitating the formulation of ethical guidelines to protect human participants. Professor Hawkins emphasized that ethical concerns during clinical trials involve consideration of both general and trial-specific ethics. For a clinical trial, the research question should be important and answerable. He further reiterated that the benefits of clinical trials should outweigh the risks involved to the participants. Research involving humans needs to take into account the ethical dimensions of the reasons for running an experiment and incorporate the proper procedural steps to ensure that the results reflect good science and are beneficial to the participants. Professor Hawkins continued by providing the history of ethics in clinical trials and provided a brief overview of abuses, scandals, and tragedies involving human participants that have led to the formulation of ethical codes and regulations for clinical trials. The Nuremberg Code of 1947 comprises principles such as informed consent, proper scientific experimentation, and fruitful results for society. In 1964, the Declaration of Helsinki introduced flexibility of the conditions of consent, which was inflexible in the Nuremberg code. During his talk, Professor Hawkins highlighted that the ethical perspective during clinical trials involves a choice between 'idealism' and 'utilitarianism'. 'Idealism' demands following the best possible ethical practices, whereas 'utilitarianism' requires maximizing the utility of clinical trials. Professor Hawkins said that, historically, there has been a low probability of response in phase I trials, and that the benefit to patients may not have been the goal. One of the major goals of phase I trials must be the therapeutic benefit rather than the identification of toxicity or dose adjustment. Investigators may therefore have an conflict of interest for reasons of securing funding, academic publicity, and career growth. Investigators should adopt a 'patient's physician first and scientist second' approach. An apparent ethical dilemma arises when physicians consider enrolling their patients into randomized clinical trials. This ethical dilemma can be overcome by a different approach called clinical equipoise, which is widely viewed as essential for an ethical randomized clinical trial. The goal of 'intervention and control' should be to maximize benefit and minimize harm. Professor Hawkins also highlighted the importance of 'informed consent' for clinical trials involving special populations such as children, fetuses, and individuals with mental disorders. The participation of vulnerable patients in clinical trials raises an ethical dilemma that needs alternatives to informed consent. Professor Hawkins concluded with the question, 'What is the right balance between perfect science, commercial considerations, and patients' preferences?'

Professor Muhammad Z. Alkawi talked about ethical regulatory requirements in research. The talk focused on research regulations in the KSA. The 'ethics' exist because of we are humans and the 'regulations' refer to the laws of land. Research guidelines in the KSA were established through a royal decree in 2001 that led to the formation of the National Committee of Bioethics (NCBE). Professor Alkawi emphasized that every research center is required to have a monitoring office and a local Ethics Committee called an institutional review board (IRB), which should be composed of a minimum of five members. The local Ethics Committee is required to conduct periodic monitoring of a research study. Before starting a clinical study, it is the responsibility of an investigator to obtain IRB approval: Without it, a study cannot be undertaken. Next, patients' consent must be obtained before starting a clinical study. Finally, all research members must follow the regulations to the letter. Professor Alkawi also outlined the guidelines for international collaborations, which require collaborative research and material transfer agreements. At the end of his talk, Professor Alkawi described various methods of monitoring clinical research, such as field visits and representation at local Ethics Committee meetings. He also suggested that a 'Research Ethics Monitoring Office' should be connected with the NCBE and responsible for enforcing research ethics during a clinical trial.

Session 3: The economic impact of phase I trials and precision medicine. The costliest step during drug discovery and development is the clinical trial because it uses humans to test the safety and efficacy of the novel therapeutics under investigation. When a trial is successful and the drug is launched, there are two major outcomes: It helps cure the patients, and it provides financial rewards for the organization that discovered and developed the drug. The aim of this session was to discuss both the costs and rewards of clinical trials, with a focus on phase I trials. The speakers of the sessions were: Professor Syed Hussain, Consultant College of Medicine and Dentistry, Plymouth University, Plymouth, UK), Mr. Saeed Noibi Health Outcomes and Epidemiology Head, Medical Affairs, Gulf Cooperation Countries; GlaxoSmithKline Plc., London, UK, Dr Michael Streit (Senior Director; Johnson \& Johnson, Cincinnati, OH, USA), Dr Yaseen Arabi (Chairman, Intensive Care Department/Medical Director, King Abdulaziz Medical City, Riyadh, KSA), Professor Julen Oyarzabal (Director, Center for Applied Medical Research, University of Navarra, Navarra, Spain), and Dr Mohamed Abumaree (Director, Stem Cells and Regenerative Medicine Department, KAIMRC).

Professor Syed Hussain talked about the impact of the clinical trials he has conducted during his career on the cost and paradigm of bladder cancer treatment. The results of these clinical trials have changed the standard of care for patients with muscle-invasive bladder cancer opting for organ-preserving treatment. He also discussed the role of neo-adjuvant chemotherapy in muscle-invasive bladder cancer, and explained that the results of ongoing clinical trials involving well-tolerated immune checkpoint inhibitors are likely to transform the outcomes of some patients with metastatic bladder cancer because of the improvement in survival and durability of response observed. In addition, Professor Hussain discussed the results of his work on split-dose cisplatin, which has extended the spectrum of patients with bladder cancer eligible for such treatment to include even those with impaired renal function. Professor Hussain concluded that the landscape of bladder cancer therapies is continuously changing, with significant improvements in durability of response and overall survival.

Mr. Saeed Noibi talked about the social and economic dimensions of phase I clinical trials. At the outset, he emphasized the need for demonstrable commitment to transparency in clinical trials that is consistent with local medical anthropology. He stated that the corollary of the extensive, costly and risky process of bringing therapies to bedside, is experimental and clinical collaboration in early phase clinical trials to aid 
timely decision making. He illustrated this with a first-in-class phenotype-guided therapy in which unique benefit of the therapy in clinical practice is being realized.

This paradigm shift to experimental medicine, he opined, has social and economic dividends due to the ecosystem of diverse expertise and skill set required. He gave examples of local collaborations between phase I units and the local community to enrich the social and economic vitality. $\mathrm{He}$ concluded his presentation with the call towards diversity in clinical trials participants.

Dr Michael Streit emphasized the importance of early study design to the development and success of phase I clinical trials. He discussed the level of complication of phase I clinical trials and their costs, which can be similar to those of phase III clinical trials. Dr Streit mentioned that competition between companies make drug discovery increasingly expensive because of the overlap of such studies. To control this, in his perspective, all companies must coordinate among one other. He also talked about micro- and macroeconomic considerations in clinical trials. Examples of microeconomic considerations include the study design (whether traditional or advanced), procedures, study population, and sites where the trial will be conducted. Regarding macroeconomic considerations, Dr Streit mentioned the productivity of pharmaceutical companies and high drug price as crucial players in the precise estimation of trial costs. Dr Streit concluded that a good selection of biomarkers, precise statistics, an efficient study design, and thorough knowledge of the published data are factors that significantly increase the chances of a successful clinical trial.

Professor Yaseen Arabi gave a talk that focused on two clinical trials he conducted on Middle East Respiratory Syndrome (MERS-CoV)-infected patients. In the first trial, Professor Arabi used the convalescent plasma of recovered donors as a passive immunotherapy for MERS-CoV infection. He showed that this methodology has a limited efficiency because of the small pool of potential donors with sufficiently high antibody titers. A second trial is currently ongoing that aims to investigate the efficacy of treatment of MERS-CoV with a combination of lopinavir/ritonavir and recombinant interferon- $\gamma$.

Professor Julen Oyarzabal discussed the role of epigenetics in cancer, and showed that alterations in the epigenetics of cancer cells can be reversed by different small molecules. $\mathrm{He}$ developed two methyltransferase inhibitors and showed the effect of these inhibitors on leukemia and B-cell lymphoma. The efficacy of these small molecules was tested both in vitro and in different animal models. The results showed that these chemical series constitute a promising therapeutic tool for unmet needs in the treatment of hematologic and solid tumors. Dr Mohamed Abumaree's main research interest is the development of stem cell-based therapies. In his talk, Dr Abumaree discussed preliminary results that showed that mesenchymal stem cells isolated from the human placenta have anticancer properties and can control cancer growth and progression in the breast. KAIMRC is considering this work for advanced testing in preparation for phase I clinical trials.

\section{Conference second day}

On the second day of the conference, preclinical drug discovery process for small molecules and cellular and immunologic therapies were discussed. The speakers gave an overview of the current most promising drug targets and latest validation approaches under consideration. In addition, several KAIMRC projects were showcased during the sessions described in the following sections.

Session 1: Bridging the gap between discovery and phase I clinical trials. Clinical trials are the most important, yet the most expensive part of drug developments are often phase II clinical trials. Any drug that fails this step typically results in a significant loss for a pharmaceutical company and reduced interest from industry overall. KAIMRC is an emerging institution planning to initiate the first phase I clinical trial in KSA especially to treat local diseases. Phase II studies have not been conducted in KSA thus far, thus pharmaceutical companies were reluctant to target this area of research. KAIMRC is seeking the first license to run this type of trials and it appears achievable. In this conference, a dedicated session was meant to bridge the gap between pre-clinical drug discoveries and phase II clinical trials that were presented by four speakers: Dr Sheraz Gul (Head of Drug Discovery, Fraunhofer, Germany), Professor Jiang Dong (Executive Director; Institute of Materia Medica, Chinese Academy of Medical Science, Beijing, China), Dr Abeer Alshukairi (Consultant in Adult infectious disease; King Faisal Specialist Hospital and Research Centre, Jeddah, KSA), and Dr Imadul Islam (Senior Scientist; King Abdullah International Medical Research Center, Riyadh, KSA).

Dr Sheraz Gul shared his drug discovery experience from the Fraunhofer (Kaiserslautern, Germany) and talked about the criteria for taking a drug discovery project through to the clinic and explained how the process can take up to five years and cost around 5-10 million US dollars. Then, Dr Gul introduced and shared his experiences of various projects including the discovery of pro-protein convertase subtilisin/kexin type 9 (PCSK9) inhibitors. PCSK9 binds to the low-density lipoprotein receptor (LDLr), such that LDL can no longer bind to it. A consequence of this is increased LDL levels in plasma. Therefore, preventing PCSK9 from binding the LDLr offers the potential to lower blood LDL levels. The mode of action of PCSK9 was puzzling for some time, due to the lack of understanding of the biology of PCSK9. Eventually, the discovery of antibodies that prevented PCSK9 binding the LDLr were made after a decade and two therapeutic agents have been approved for clinical use.

Professor Jiang Dong talked about berberine, which is a quaternary ammonium salt of the proto-berberine group of benzylisoquinoline alkaloids. This chemical is extracted from Chinese plants, and has gained the attention of the scientific community due to its ability to treat arrhythmia, diabetes, hyperlipidemia and cancer. Professor Dong and colleagues have identified the crystal structure of berberine and elucidated some of its mechanism of action in relation to the LDL receptor. The drug is being used as an anti-diabetic agent, and has provided insights into new potential targets in this area of research.

Dr Abeer Alshukairi presented the symptoms of MERS-CoV and challenges faced during elucidation of the biology of MERS-CoV infection in humans. Dr Alshukairi showed how MERS-CoV induces an elevation of cluster of differentiation (CD) 4 T-cells in hospitalized intensive care unit 
patients, whereas CD8 levels vary among patients. This finding has added to our understanding of the effect of MERS-CoV, and revealed a novel target for pharmaceutical companies. In addition, it has allowed us to understand the reaction of the immune system to MERS-CoV infection.

Dr Imadul Islam discussed serendipity in drug discovery, and showed how certain drugs, including some of the most famous medicines, were discovered by sheer chance. In addition, Dr Islam presented some of his work on the development of an anti-breast cancer drug. This drug is moving toward preclinical studies in animals, with good potential to reach the market.

Following the presentations, a panel discussion was initiated to give recommendations and tips to KAIMRC for its journey toward the initiation of a preclinical drug discovery program and phase I clinical trials. Guidance was also given on how to exploit beneficial resources around KAIMRC. The discussion included advice on how to attract companies to contribute to the initiation of clinical trials.

Session 2: Target and hit identification. The most challenging and important part of therapeutics discovery is to identify the target that is the cause of the disease. It is crucial that this step is correct because it represents the cornerstone of a drug discovery project. The association of the target to the disease needs to be validated by number of approaches, including genomics and in vitro and in vivo animal testing using multiple techniques. Once the target is identified, it will be used to identify hits in the form of small molecules, antibodies, mRNAs, and others agents that either inhibit or activate the target to modulate its function in the disease under investigation. This session addressed these aspects of therapeutics discovery with the participation of the following speakers: Dr Michael Streit (Senior Director; Johnson \& Johnson), Dr Rana Rais (Assistant Professor, Johns Hopkins School of Medicine, Baltimore, MD, USA) and Dr Mohamed Boudjelal and Dr Zeyad Alehaideb from KAIMRC.

Dr Michael Streit talked about genomic data mining and target identification. He also shed light on reverse translational research and genomic data as valuable tools to identify disease targets. Genomic data mining and target identification involve computational science, which may be compound-, patient- or population-centric. Target identification and selection considers disease progression, druggability, and competition. $\mathrm{He}$ presented a case study of prostate cancer using three attributes: Disease-specific, small-molecule, and cell-surface targets for prostate cancer. Dr Streit concluded that genomic data mining is a powerful tool to initiate target identification research. One drawback is the big and messy nature of the data: Sophisticated algorithms are required to extract the relevant data. Also, he stated that genomic data should match with biologic data to establish the relationship between target and disease.

Dr Rana Rais presented a well-known glutamine antagonist, 6-Diazo-5-oxo-L-norleucine (DON), which has been used in preclinical and clinical studies. Her laboratory has synthesized analogs of DON to improve its efficacy and help alleviate its associated gastrointestinal toxicity. She shared data showing the plasma and liver stability of these analogs, and the specific release of DON in tumor cells. In a head-to-head toxicity study that also examined tissue distribution, she showed that DON caused much more serious adverse effects compared with the DON prodrug, and that significantly less DON was released in abdominal tissues using the novel compounds. Lastly, Dr Rais stated that the novel compounds underwent specific uptake and bioactivation in the brain, providing more DON at the target site (brain) and less in the periphery (plasma). There was an almost 10 -fold improvement in the brain/plasma ratio compared with DON in monkeys and pigs.

As an example of preclinical drug discovery efforts at KAIMRC, Dr Mohamed Boudjelal described a new, naturally transformed cell line, KAIMRC1, isolated from the breast tumor of an Arab woman aged 62 years who was suffering from ductal breast carcinoma. The cell line was characterized for its biologic and molecular markers, induction of mitogen-activated protein kinase signaling, and response to different commercially available drugs. Dr Boudjelal stated that KAIMRC1 breast cancer cell line is estrogen receptor/progesterone receptor (ER/PR)-positive and human epidermal growth factor receptor 2 (HER-2)-negative. The epithelial nature of this cell was confirmed by flow cytometry using epithelial cell markers. Its morphology and genetic profile were also described. This cell line provides a source to identify new breast cancer targets that lead to its natural transformation. Also, it can be used to develop phenotypic assays to screen for novel anti-proliferation hits.

Dr Zeyad Alehaideb presented his work on the effect of an extract of a local shrub, Calligonum comosum (Al'Ar'ta in Arabic), on the migration and viability of the triple-negative MDA-MB-231 human breast cancer cell line. The effect of this shrub has never before been evaluated on the aforementioned aggressive breast cancer cell line, and this is the first study of its kind. The methanolic extract of the shrub showed a dose-dependent inhibition of migration and induced cell death compared with untreated cells. Also, Dr Alehaideb discussed the changes in cell morphology and migration. He concluded that Calligonum comosum has the potential to prevent the progression of triple-negative breast cancer (TNBC), and more work is underway to study the composition and mode of action of Calligonum comosum extract.

Session 3: Nanomedicine, formulations and natural products. In the last decade, nanomedicine research, which is the application of nanotechnology to medicine, has become one of the most promising areas of biomedical research to improve the general and site-specific delivery of therapeutic and diagnostic agents. Advances in nanomedicine may provide numerous improvements to drug efficacy, including bioavailability, targeting ability, and safety. The speakers in this session were: Professor Thierry Vandamme (University of Strasbourg, Strasbourg, France), Dr Rabih O Alkaisy (Associate Professor; King Saud Bin Abdulaziz University, KSA), and Dr Bahauddeen Alrfaei (Scientist; Stem Cell Scientist, KAIMRC).

Professor Thierry Vandamme delivered a talk about nanoemulsions as a strategic platform for innovative drug delivery systems. Nanoemulsions are emulsions that are manufactured on a nanoscale level to improve the delivery of active pharmaceutical ingredients. Nanoemulsions constitute an advanced mode of drug delivery, which has been developed to overcome the major drawbacks associated with conventional drug delivery systems. In his talk, he gave a basic overview 
of nanoemulsion formulation, preparation, characterization, evaluation, and applications. As a drug delivery system, nanoemulsions may enhance the therapeutic efficacy of drugs and minimize their adverse effects and toxicities. Major applications include biomedical imaging, treatment of infection of the reticuloendothelial system, enzyme replacement therapy in the liver, treatment of cancer, and vaccination.

Dr Rabih O Alkaisy discussed the future applications of functional molecular crystals in medicine. Organic molecular crystals were long considered a 'chemical cemetery', with limited use in chemistry, material science, or medicine. By synthesizing photochemically active small molecules and co-precipitating them from water/surfactant, Dr Alkaisy and colleagues were able to fabricate uniformly shaped molecular crystals with useful function that extend to medical applications such as microrobots and microcapsules for drug delivery. Dr Alkaisy showed the application of this method to deliver doxorubicin.

Dr Bahauddeen Alrfaei's talk focused on the relationship between the deficiency of 2'-5'-oligoadenylate synthetaselike (OASL) and the migration ability of macrophages and lymphocytes. OASL is thought to play a role in viral infection. OASL-deficient patient blood samples showed reduced levels of both granulocyte-macrophage colony stimulating factor and Vascular endothelial growth factor compared with blood samples from healthy volunteers. Dr Alrefai's data also showed that OASL deficiency results in reduced migration of macrophages and lymphocytes compared with their normal counterparts. The results from his laboratory indicate that OSAL-deficient patients may be more susceptible to non-viral infections than healthy individuals. These changes may explain the partial developmental delay in OASL-deficient patients' symptoms.

Session 4: Small molecule drugs versus biologics (antibodies and chimeric antigen receptor T-cell therapy). The last session of the conference focused on the topic of adoptive T-cell therapies. Cancer immunotherapy is a treatment that uses patients' immune systems to combat cancer. Adoptive cell therapy, a form of cancer immunotherapy, involves enrichment and expansion of immune cells ex vivo, followed by their infusion into the patient. A relatively novel immunotherapy approach, chimeric antigen receptor T-cell therapy (CART), has emerged in the field of cancer immunotherapy. CART is a type of adoptive cell therapy that was initially restricted to small clinical trials mainly involving patients with advanced blood cancer. However, because of the significant outcomes of this treatment, researchers are exploring its efficacy on other types of cancer. The session's speakers were: Professor Robert Hawkins (Chair of Medical Oncology; University of Manchester and Christie Hospital, Manchester, UK), Professor Jean-Marc A. Nabholtz (represented by Dr Khalid Alsalh, Department of Medicine, Division of Onco-Hematology, King Saud University Medical City, KSA), Dr AbdulWajid Abdulwajid (Senior Director of Vaccine Process and Manufacturing Sciences; Agenus Inc., Berkeley, CA, USA) and Dr Ensaf Al-Hujaily and Ms. Balkese Alhammad from KAIMRC.

Professor Robert Hawkins discussed an exciting area of immunotherapy in his talk 'Developing Adoptive T-cell Therapy for Cancer: Achievements and Challenges to Treat
Solid Tumors'. Professor Hawkins, an expert in the field, presented preclinical models and clinical experience indicating the efficacy of adoptive T-cell therapy. Professor Hawkins showed promising results of phase I and -II clinical trials involving ex vivo stimulation of autologous tumor-infiltrating T-cells (TIL) to treat solid tumors, including melanoma. He emphasized that successful adoptive T-cell therapy is dependent on the ability of the infused lymphocytes to travel to the tumor site and mediate tumor destruction. As a type of adoptive T-cell therapy, CART can be radically effective in targeting CD19-positive B-cell malignancies. However, this approach is subject to many challenges, including: The identification of suitable targets, tumor antigen heterogeneity, 'on-target and offtumor' toxicity, and difficulties in engineering immune cells to make them more effective. During his talk, he described preclinical and clinical experience of testing natural T-cells, T-cells engineered with T-cell receptors, and CART cells. Professor Hawkins is using preclinical models to examine the potential toxicity of this treatment. In addition, he shared his experience of developing clinically applicable systems to reduce the toxicity of supportive therapy and control the function of T-cells in vivo.

On behalf of Professor Nabholtz, Dr Khalid Alsaleh presented a talk entitled 'Shifting Paradigms in the Development of Biologic Therapies in Breast Cancer'. He gave an overview of the present challenges facing the treatment of cancer and breast cancer. Knowledge of cancer biology is presently exploding, with the identification of multiple biologic targets leading to the potential availability of numerous targeted therapies in the clinic; however, the translation to patient care remains sketchy. This paradox is explained by the present principles of breast cancer therapy and strategies for the development of new biologic modifiers, which are still mostly based on a limited number of biomarkers [hormonal receptors (HRs), proliferation markers, and HER-2 gene status]. These biomarkers have a well-established prognostic value and some crude predictive value, in particular for HER-2 and HRs. Consequently, this has led to simplistic anatomo-pathologic classifications defining large subgroups of patients, resulting in the necessity to proceed, in research and development, with large-scale clinical trials seeking modest differences when comparing new targeted therapies to standard treatments. This approach can be defined as developing 'targeted therapies without specific targets', and fails to address the biologic complexity of breast cancer and rapid adaptation of tumors to various therapies. Breast cancer is a dynamic disease involving the regulation of DNA expression; hence, there is a need to rethink strategies for the development of biologic modifiers, focusing on the use of advanced technologies (quantum optics, methylation of DNA with next generation sequencing, bioinformatics with supercomputers) to allow real-time repetitive analyses of individual biologic profiles from liquid biopsies. Such an approach could lead to the definition of individual biologic profiles at $\mathrm{n}$ variables (hundreds or more instead of four at the present time, i.e. the estrogen and progesterone receptors, HER-2 gene status, and a proliferation marker). The need for 'correlations' between theses 'profiles' and the efficacy of/resistance to therapies will require access to powerful bioinformatics processes and adapted software. The ultimate aim is to build 'individual dynamic blood tumor mappings' leading to a 'Breast Cancer 
Translational Platform', which could impact greatly the future management of breast cancer patients and the global development of new biologic therapies.

Dr AbdulWajid Abdulwajid delivered a talk entitled 'Challenges of Antibody Generation, Clone Development followed by Manufacturing of Antibodies'. During his talk, he discussed the process of antibody cloning, evaluation, and the selection of lead clones. The selection of lead cell lines is based on specific criteria, including the quality of the clones, cell line stability, and antibody titer. Furthermore, the antibodies are characterized using different techniques; eventually a Good Manufacturing Practice (GMP)-grade antibody is tested and released for clinical trials.

Dr Ensaf Al-Hujaily presented a talk about CART cells and multiple myeloma. Although there have been many advances in the treatment of multiple myeloma, it is considered an incurable disease: The high rate of relapse, usually associated with refractory disease, necessitates the development of novel treatments. Dr Al-Hujaily showed that CART is a promising treatment modality for multiple myeloma based on the findings of a comparative clinical study using CART cells. However, she warned of the adverse effects of CART by addressing the caveats of using each treatment. Dr Al-Hujaily stated that the main adverse effects were cytokine release syndrome and 'on-target off-tumor toxicity'.

The last speaker of this conference was Ms. Balkese Alhammad. Ms. Alhammad gave a talk about the characterization of breast cancer neo-antigens and their recognition by tumor-infiltrating lymphocytes. Ms. Alhammad gave an overview of the TNBC subtype, in which she stated that most of the targeted therapies used to treat breast cancer cannot treat TNBC patients. Hence, there is a massive need to explore novel treatment strategies for TNBC patients. Immunotherapy is the strategy most likely to cure TNBC: Adaptive cell transfer using TILs has shown positive outcomes in other immunogenic tumors, and therefore it should be applied for TNBC.

\section{Conference recommendations: Proceeding extracts}

1. Over the years, the goals of phase-I clinical trials have moved away from classical objectives by merging phase-I and -II trials, creating larger phase-I trials and basket trials. The success of these trials is largely dependent on both preclinical development and clinical trial design.

2. Phase-I clinical trials should not only be designed to test toxicity or dose adjustment, but must also consider the therapeutic benefit to the patient. The investigators should rise above personal conflicts of interest related to securing funding, academic publicity, and career growth, and adopt a 'patient's physician first and scientist second' approach.

3. Apart from solid scientific experience and infrastructure, trust, discovery, and decision-making are key to the successful establishment of phase-I clinical trials. It is essential that society should be convinced about the beneficial contribution of these trials to society and medical anthropology.

4. During clinical trials, investigators need to choose between 'idealism', which demands following the best possible ethical practices, and 'utilitarianism', which requires the maximum utility of clinical trials.

5. As a result of overlapping studies, competition between companies leads to a very expensive drug discovery program. This may be controlled by the coordipnation of these companies.

6. A shift from a one-size-fits-all approach to a personalized medicine strategy has increased the focus of drug development programs on small molecular inhibitors.

7. To enhance collaboration between academia and industry, it is essential to have an 'Early Drug Development Unit' that will strengthen the program and resources investment.

8. Precision medicine requires characterization of genomic and immune changes to tailor therapies to achieve the best response and highest safety for the patient.

9. Strategies to develop biologic modifiers, involving advanced technologies such as quantum optics, methylation of DNA with next generation sequencing, and bioinformatics with supercomputers, may be useful to characterize dynamic diseases such as breast cancer.

10. A successful CART approach, that can effectively target CD19-positive B-cell malignancies, faces challenges including the identification of suitable targets, tumor antigen heterogeneity, 'on-target off-tumor' toxicity, and difficulties in engineering immune cells to make them more effective.

11. A good understanding of a disease, in combination with the use of suitable techniques that include phenotypic assays and a proper drug delivery system, is key to a successful drug discovery program. 